\title{
marges Marges
}

revue d'art contemporain Revue d'art contemporain

$08 \mid 2008$

L'art à l'heure de la société de services

\section{Économie du tertiaire / Économie de l'art. La place du multiple}

Third Sector Economies / Art Economy. The Question of the Multiple

Océane Delleaux

\section{(2) OpenEdition}

Journals

Édition électronique

URL : http://journals.openedition.org/marges/569

DOI : $10.4000 /$ marges.569

ISSN : 2416-8742

Éditeur

Presses universitaires de Vincennes

Édition imprimée

Date de publication : 15 octobre 2008

Pagination : 21-32

ISBN : $978-2-84292-252-8$

ISSN : $1767-7114$

Référence électronique

Océane Delleaux, «Économie du tertiaire / Économie de l'art. La place du multiple », Marges [En ligne], 08 | 2008, mis en ligne le 15 octobre 2009, consulté le 19 avril 2019. URL : http://

journals.openedition.org/marges/569; DOI : 10.4000/marges.569 


\section{Économie du tertiaire/Économie de l'art. La place du multiple}

Sous l'effet conjugué du développement du secteur tertiaire, des progrès technologiques et de la mondialisation, on assiste ces quinze dernières années à l'émergence d'une nouvelle ère économique, tournée de plus en plus vers la connaissance et le capital immatériel. Sur le terrain artistique, cette immatérialisation au sein des échanges humains et commerciaux se traduit notamment par le recours aux nouvelles technologies, la création de dispositifs interactifs et conviviaux, sans production d'objets spécifiques. Elle s'accompagne bien souvent du souci des artistes d'inscrire une pensée économique dans leur projet.

Contre toute attente, ce type de préoccupation est intimement lié à la pratique du multiple, c'est-à-dire aux objets tridimensionnels d'artistes, destinés à être tirés en le plus grand nombre d'exemplaires possible. Ainsi, lorsqu'en 1998 Nicolas Bourriaud traite des productions "processuelles ou comportementales » qui lui sont contemporaines, il n'omet pas de citer les rats de Maurizio Cattelan, nourris avec du fromage avant d'être vendus comme multiples (I Found My Love in Portofino, 1994'1). La pratique du multiple permet en effet de prendre en compte les conditions de production et de distribution des œuvres et donc d'introduire une dimension économique dans la pratique artistique. Car si l'économie de service suppose une activité qui n'est pas directement rattachée à la production de biens, elle y est de toute évidence généralement associée. Dans ce contexte, on s'interrogera sur la capacité du multiple à intégrer des structures artistiques qui placent les services au centre de leur problématique. Le but est de montrer comment l'artiste peut proposer une œuvre matérielle, qui plus est multipliée et à la disposition du spectateur, au sein d'une œuvre plus globale et immatérielle. L'exemple de la société Soussan Ltd Fournisseur des musées, appuiera notre argumentation. Il permettra de clarifier la place du multiple au sein d'une économie qui veut appartenir au domaine du tertiaire mais qui n'en relève pas moins de celui de l'art.

\author{
${ }^{1}$ Nicolas Bourriaud, \\ Esthétique relationnelle, \\ Dijon, Les Presses \\ du Réel, 1998, p. 7-8.
}


2 identifiée par le logo.

Soussan Ltd Fournisseur des musées

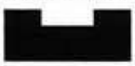

3 Sylvain Soussan, entretien avec Emmanuelle Chérel, «Conversation avec Sylvain Soussan P.D.G. de Soussan Ltd. et conseiller technique du musée des nuages ", Soussan Ltd., Pougues-les-Eaux, Parc Saint-Léger, centre d'art contemporain, 2001, pages 27-28.

4 ibid., p. 11.

5 Sylvain Soussan, entretien avec l'auteur, Paris, 16 septembre 2005.

${ }^{6}$ L'artiste ne signe pas ses produits et fournit uniquement une facture en guise de certificat d'authenticité.

\section{Soussan Ltd : une entreprise au service de l'art}

C'est en 1989 que l'artiste Sylvain Soussan annonce la reprise, en tant que président-directeur général, de la société Soussan Ltd à Sceaux. Il s'agit d'une entreprise familiale fondée par son ancêtre Marcel Soussan qui aurait inventé le thermohygrographe à destination du marché de l'art.

Cette société ${ }^{2}$ vise à établir des relations commerciales avec les acteurs de l'art contemporain. Aussi propose-t-elle aux musées et aux particuliers une production de matériels et une offre de services : équipement destiné à l'exposition ou à la conservation, expositions livrées "clé-en-main », services de restauration, manutention et sécurité, mise en valeur des collections ou encore organisation de concours.

Soussan Ltd trouve notamment sa visibilité grâce à son site Internet www.soussan-ltd.com, mis en service en 2001, avec les conseils du graphiste Éric Arlix. Ce site s'organise en trois rubriques: produits, services et mécénat. Chaque rubrique contient l'ensemble des références Soussan, présentées en quelques lignes sur un mode publicitaire. Trois icônes permettent l'accès à un deuxième niveau d'information: outil de visualisation (démo, application flash, diaporama), résumé des références (fiches mémo) et complément de documentation. Afin de toucher un large public, Sylvain Soussan souligne d'ailleurs l'emploi de "supports habituellement mis en place par la publicité » pour montrer des œuvres: « c'est un travail de propagande pour l'art, il s'agit d'occuper le terrain ${ }^{3} »$.

On peut appliquer cette réflexion aux catalogues de Soussan Ltd, à l'image de celui paru pour l'exposition « Soussan: entreprise culturelle » à l'Écomusée de Savigny-le-Temple en 1990-91. L'ouvrage présente la société, son histoire et ses finalités, accompagnés d'un organigramme de l'entreprise et d'une liste des produits disponibles. Dans un entretien avec Emmanuelle Chérel en 2001, Sylvain Soussan rappelle: «En tant qu'industriel, j'ai tenu un langage industriel, je n'ai pas publié des catalogues d'artistes mais des catalogues de produits 4 . " Mais au cours d'un entretien en septembre 2005, il nous confie qu'il considère aussi ce catalogue comme un livre d'artiste5. De ce fait, cette publication associe la fonction de catalogue de vente à celle de catalogue d'art. L'artiste met en place une fiction basée sur le langage de l'entreprise, sur lequel il greffe un certain nombre d'éléments susceptibles de renvoyer au champ artistique. Par exemple, les numéros de référence des produits listés à l'intérieur sont en réalité attribués après vente et servent à leur authentification sur le marché de l'art ${ }^{6}$. 
Au premier abord, Soussan Ltd semble se présenter comme une véritable société du tertiaire, de la proposition de produits, de services et d'un mécénat, jusque dans sa communication où les catalogues et le site Internet remplissent d'abord le rôle de diffuseurs. D'emblée, on s'aperçoit que Soussan Ltd implique une production de matériels comme le montre l'analyse des produits infra. On verra aussi que les deux autres secteurs d'activité de l'entreprise, à savoir les services et le mécénat, ne sont pas uniquement basés sur la mise en place de dispositifs immatériels, comme toutes les sociétés de services, d'ailleurs.

\section{Soussan Ltd : de la rareté des produits}

Commençons par les produits. Ce sont les différents biens de Soussan Ltd, fabriqués en général en coopération avec une société industrielle et dont la consommation est laissée à l'appréciation de l'acquéreur. Ils s'inscrivent dans la ligne directrice de l'artiste, à savoir « tenir un discours un peu critique vis-à-vis de la production artisanale institutionnelle en montrant que ses excès conduisent à un dialogue entre nous, à un espace sans surprise pour les uns et sans échange pour les autres, à des "private jokes" 7 ». Ils sont disponibles à la vente auprès de l'artiste, en particulier sur le site Internet de Soussan Ltd:

- Conservez votre instinct de conservation est un conservateur liquide, vendu dans un flacon longue conservation. Ce «produit miracle ${ }^{8}$ " est censé agir sur les facteurs d'altération des œuvres d'art afin de remédier aux craintes des professionnels de l'art. Il est présenté et testé dans l'espace d'accrochage de l'Abbaye SaintAndré, Centre d'art contemporain de Meymac en 1990. Quelques exemplaires sont fabriqués et vendus à la galerie Le Sous-Sol à Paris pour 150 francs (22,87 euros).

- L'Hygromètre est un appareil de mesure du degré d'humidité de l'air, fabriqué par la société Jules Richard et personnalisable selon un choix de couleurs, matériaux d'habillage, décors ou accessoirisation spécifique. Cette customisation de l'objet renforce l'idée selon laquelle les diagrammes réalisés à partir de chaque hygromètre sont une partie de l'essence des œuvres qu'il contribue à préserver. L'option d'une location permet également la réalisation de diagrammes certifiés par Soussan Ltd. Quatre modèles (une pièce unique de chaque) ont été réalisés depuis 1990.

- Les Outils-faits-main-pour-votre-main sont diffusés par Soussan Ltd mais conçus par l'artiste Christian Viallard, à l'issue de l'exposition « Fournir les musées, un travail d'artistes » à l'École nationale
7 Sylvain Soussan, entretien avec Emmanuelle Chérel, op. cit., p. 27.

8 Sylvain Soussan, entretien avec l'auteur, Paris, 2005. 
9 D'autres outils personnalisés ont été produits tels qu'un pinceau, un rouleau et un mètre.

${ }^{10}$ Ces extincteurs ont été présentés lors d'une exposition au Spazio Erasmus à Milan (26 février - 23 mars 1997), à la Galleria d'Arte Caserte à Naples

(5 juillet-5 août 1997) et à la galerie Le Sous-sol (25 avril-28 mai 1998).

${ }^{11}$ La peinture a été appliquée sur les murs de la galerie le Sous-Sol à Paris par l'artiste Bernard Brunon en 1994. Soussan Ltd. a aussi fourni la peinture nécessaire

à la réalisation de l'Espace blanc par Roberto Martinez au Centre d'art contemporain de Rueil-Malmaison en 1997.

12 L'utilisateur reçoit un imprimé qu'il doit insérer dans l'envoi d'invitations suivant la première application de blanc. L'imprimé comprend la signature ou le logo du collectionneur, ainsi que la mention «Blanc par Soussan Ltd, peinture professionnelle pour les expositions... pour que le fond retrouve la forme».

13 Robert Filliou, Poussière de poussière de l'effet... , 1977. Boîte en carton avec chiffon et polaroïd. Paris, Édition La Boutique Aberrante, Daniel Spoerri. 100 ex. des arts décoratifs de Limoges en juin 1995. On peut notamment acheter un tournevis en bronze, une « édition illimitée commençant par un tirage de trois exemplaires » à 2500 francs (381,12 euros), un marteau chromé « usiné à la main selon une méthode exclusive d’un exemplaire à l'autre » à 6000 francs ( 914,69 euros) ou encore un Marteau-en-cristal-de-roche qui incite à la prudence car exécuté à la commande dans des gemmes différentes par un artisan cristallier9. - Des Extincteurs sont aussi proposés depuis $1997^{\mathbf{1 0}}$. Ils consistent en une gamme de cinq "rouge incendie » à appliquer sur des extincteurs avec un habillage graphique à choisir parmi cinq propositions. Les extincteurs deviennent des productions à part entière de l'exposition qu'ils concourent à révéler tout en respectant la réglementation de leurs modèles et leur entretien par une société prestataire au choix du client. Trois ont été peints en usine dans une chaîne de fabrication, avec le mode d'emploi appliqué au dos, selon trois teintes différentes variant du rouge au rose. Deux d'entre eux portent le label jaune du fabricant (ici Bloc-feu) qui certifie leur conformité aux règles de sécurité d'incendie et la possibilité de les installer en galerie. Un seul extincteur a été vendu.

- Le Blanc professionnel, pour que le fond retrouve la forme est une peinture murale sélectionnée à la demande de Soussan Ltd par l'artiste coloriste Daniel Walravens parmi les peintures murales existantes et dont l'application est confiée à l'artiste Bernard Brunon (THAT'S PAINTING Productions). La peinture a été spécialement utilisée pour plusieurs expositions ${ }^{11}$. Ce qui fait d'elle une œuvre in situ dans le cube blanc de la galerie, une version industrialisée du Carré blanc sur fond blanc de Malévitch qui révèle a contrario le sentiment d'objet par son rapprochement avec d'autres œuvres. Le blanc représente selon l'artiste un label, une charte de qualité pour la peinture. Le principe est de chercher une peinture qui corresponde à cette charte et de trouver une collection intéressée par son achat. Depuis 2002, le site Internet offre 150 mètres carrés ou 15 kg de peinture blanche en remerciement pour toute proposition de partenariat avec une galerie ${ }^{12}$.

- Enfin, Technique de surface renvoie au matériel d'entretien estampillé Soussan Ltd (lot de toiles lavantes, brosses et balais pour 300 euros, chariot de nettoyage, équipé et prêt à l'emploi pour 900 euros et nettoyeurs automatiques avec sous-traitance). La performance-nettoyage ne menace pas le système de contemplation et de valorisation de l'œuvre et de son commerce, à l'instar des chiffons à poussière de Robert Filliou ${ }^{13}$, mais plutôt un moyen d'insertion des usages au sein des œuvres d'art. Fin 2003, le robot nettoyant est mis en service au musée du Louvre ainsi que pour 

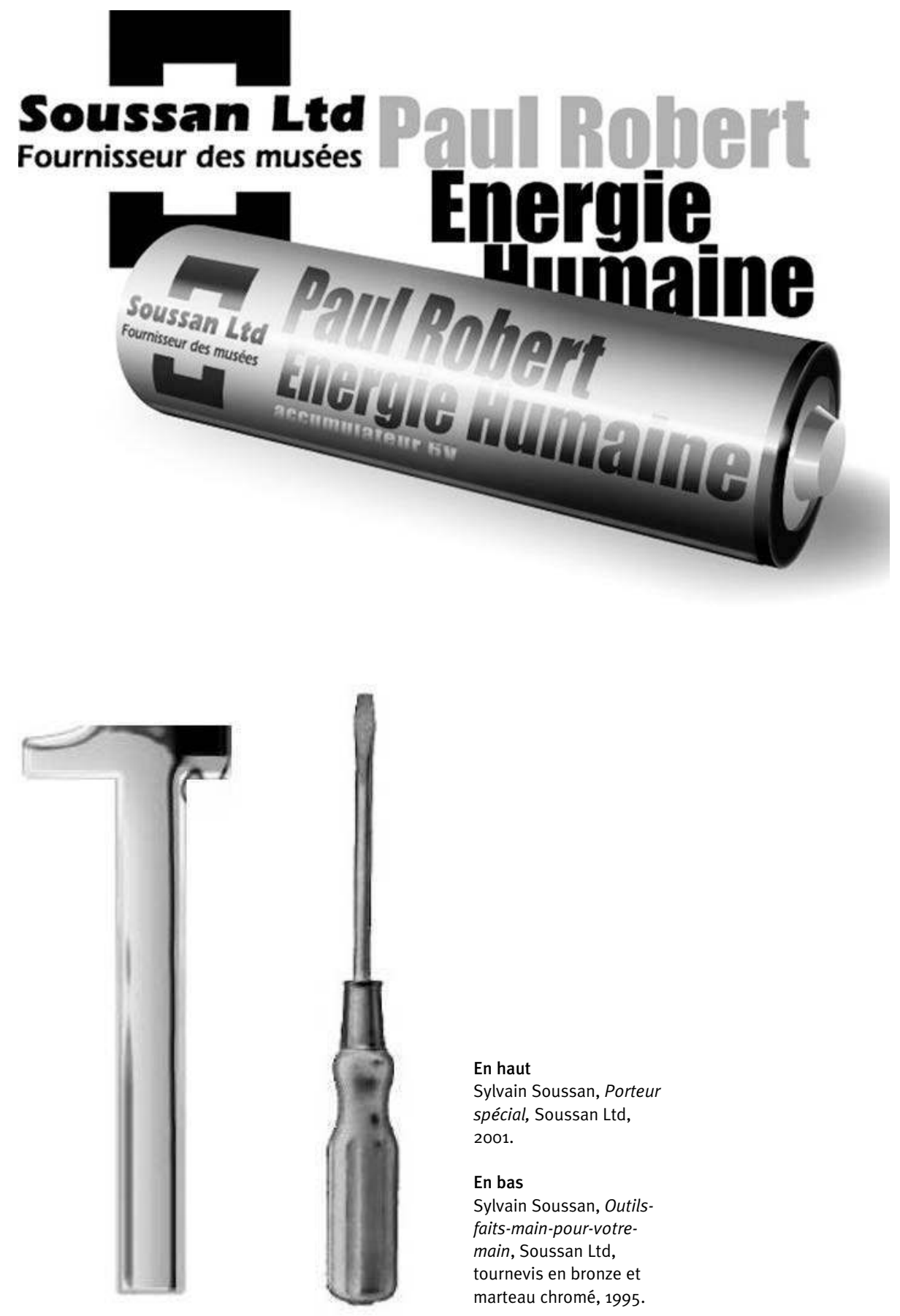

En haut

Sylvain Soussan, Porteur spécial, Soussan Ltd, 2001.

En bas

Sylvain Soussan, Outilsfaits-main-pour-votremain, Soussan Ltd, tournevis en bronze et marteau chromé, 1995. 
${ }^{14}$ Sylvain Soussan, entretien avec Emmanuelle Chérel, op. cit., p. 27.

15 Sylvain Soussan, entretien avec l'auteur, Paris, 16 septembre 2005. l'exposition "Ma petite entreprise » à l'Abbaye Saint-André de Meymac. Il s'agit d'une pièce unique. Une vidéo de démonstration des produits est aussi disponible au prix de 60 euros.

Tous les produits de Sylvain Soussan sont destinés à être utilisés dans leur fonction initiale. Au regard des applications présentées en exposition, les collectionneurs et les institutions jouent le jeu. Les exemples précédents montrent que l'artiste s'adapte aux codes et aux fonctionnements d'une entreprise, au-delà de la simple simulation. Aussi refuse-t-il, à juste titre, le terme d' " entreprise fictive ${ }^{14}$ ». Puisque ces produits consommables jusqu'à l'usure, voire périssables, sont de surcroît fabriqués en plusieurs exemplaires, on peut également s'interroger sur leur capacité à s'inscrire dans la pratique plus spécifique du multiple d'artiste.

Pour autant, si une certaine confusion sur le statut des objets est entretenue, ceux-ci s'inscrivent dans un contexte artistique, qu'ils soient uniques ou multiples. Bien que labellisés « Soussan Ltd », ils ont une dimension artistique comme le montre leur processus de communication: "objets de collection au service des collectionneurs » (outils), « œuvres qui ne ressemblent pas à des œuvres » (extincteurs), " pièces de collection au service des œuvres d'art » (hygromètres), " l'esthétique [...] au rendez-vous » (toiles et brosses) ou encore " potion magique synthétisée en une formule unique » (conservateurs). Leur mise en fonction ne signifie donc pas leur disparition en tant qu'œuvres. Au contraire elle finalise le processus de leur association à un cadre artistique qui interagit avec elles.

Sylvain Soussan parvient non seulement à simuler les activités d'une entreprise mais aussi à en intégrer certains modes de fonctionnement jusqu'à composer avec de véritables industries; les œuvres-produits qui en résultent sont loin de suivre les volumes d'édition de leurs homologues industriels. L'analyse des exemples précédents montre en effet que peu d'entre elles réussissent à dépasser le statut de prototype ou de la définition la plus ouverte du multiple, c'est-à-dire une œuvre originale tirée à trois exemplaires minimum. D'ailleurs, la plupart des produits sont fabriqués à la demande. À cet égard, l'artiste relate les difficultés de convaincre à chaque fois les industriels ${ }^{15}$. Car au-delà de la résistance de certains à l'art contemporain, il importe pour la grande majorité d'entre eux d'avoir la garantie d'une demande s'adaptant au plus grand nombre.

De ce fait, le multiple n'est pas nécessairement là où on l'attend le plus au sein d'une société du tertiaire, en l'occurrence dans l'offre des produits. Les activités de service, censées représenter une part 
au moins aussi importante dans l'entreprise concernée, sont en mesure de montrer d'autres types de propositions esthétiques multipliées.

\section{Soussan Ltd: des éditions de services}

Les services sont les prestations fournies par Soussan Ltd à titre onéreux, en collaboration avec une société industrielle. Souvent produits à la demande, ils sont ensuite vendus par correspondance, via le site de l'entreprise. La maintenance des productions est effectuée par une société prestataire recommandée par Soussan Ltd ou choisie par l'acquéreur.

- Fountain 1997 est un distributeur automatique de boissons qui maintient le rapport du ready-made à l'industrie pour le service des professionnels de l'art. Vendu 12200 euros, il est conçu comme un support d'exposition: la façade éclairante est offerte à un artiste (Claude Closky, Philippe Mayaux, Hans-Jörg Mayer, Istvan Balogh et Virginie Barré). Ces distributeurs occupent divers espaces tels que la Délégation aux Arts Plastiques à Paris, le bar du Palais de Tokyo, le Centre Pompidou ou encore la piscine de Fontenay-le-Comte.

- Mouvement artistique ${ }^{T M}$ est un service de manutention des œuvres d'art qui transporte les œuvres en présence des visiteurs au rythme de quelques centimètres par jour, grâce à des transpalettes et des gerbeurs. L'intervention n'est pas annoncée par un cartel mais par une main courante posée sur l'œuvre. Cette performance permet au visiteur de s'imprégner de l'œuvre par rapport à l'espace qui l'entoure. Ce service est par exemple utilisé pour le transport d'une œuvre de Martin Kippenberger à la vitesse de 32,726 centimètres par jour, du 21 novembre 1997 au 3 février 1998 au Mamco de Genève.

- Fantômes est un bordereau mis en place à partir de 1999. Déposé à l'emplacement laissé vacant par une œuvre enlevée provisoirement d'un espace d'exposition, il montre "le travail d'artistes vivants ${ }^{16}$ ", c'est-à-dire un ensemble de portraits d'individus issu de la banque d'images Grore Images et dont les droits de reproduction peuvent être achetés. Les fiches à remplir et à signer par le musée reprennent les garanties des cartels tout en affichant un statut artistique obtenu par les réappropriations successives de Soussan Ltd et des acheteurs. Le fichier du bordereau à imprimer est adressé par e-mail pour 50 euros, l'impression en tirage unique du bordereau expédiée par la poste pour 100 euros.

- L'Isoloir est un isoloir pour téléphones mobiles à poser dans les espaces d'exposition, avec différents matériaux et formes possibles, ainsi qu'un logo prêt à être confié à une société de lettrage
16 Une présentation a eu lieu dans le hall de la Délégation aux Arts Plastiques, du 4 au 24 février 1999. Soussan Ltd, Sceaux [en ligne]. Disponible sur: http://www.soussancom .com/présentation services.htm 
${ }^{17}$ IT2M, Isoloir pour Téléphones Mobiles Mobile (monté sur châssis roulant) est proposé à titre exceptionnel (devis et plans sur demande).

18 Une présentation a été effectuée lors d'un marathon disputé par Paul Robert au cours des Championnats de France en 2001. et signalétique. Il vise à instaurer de nouveaux dialogues dans le public sur les œuvres exposées, à l'image de celui installé pour l'exposition «Carnet d'adresse: un critique, une œuvre, un artiste » au Musée de Louviers en $2000^{17}$.

- Le Diable est un poste de présentation et de diffusion de documents multimédia, en l'occurrence d'œuvres numériques ou de programmes de Soussan Ltd, vendu pour 5300 euros (livraison, installation et mise en service inclus, maintenance à assurer par un tiers). Le Diable devient un support d'exposition tout en étant inséparable de la diffusion d'œuvres, qu'elles soient de Sylvain Soussan ou d'autrui. Le Diable existe en trois versions: "JeVoisLeDiable ", « JeSuisLeDiable » et « J'ÉcouteLeDiable » qui diffusent respectivement des programmes multimédia par l'intermédiaire d'un écran vidéo (avec connexion Internet et lecteur de CD-Rom) et d'un projecteur de DVD, ainsi que des programmes musicaux via une sono. Il est possible de se procurer le CD promotionnel réalisé pour l'exposition " LMX Étape 2 » au FRAC PACA en 2000-01 (diffusion quotidienne et en continu par le poste de la projection publicitaire "Soussan Ltd exercice 2000 »).

- Les Porteurs d'eau sont des fontaines mobiles alimentées en eau de source réfrigérée, tempérée ou chaude et dont l'habillage est réalisé sur des objets manufacturés. Une location-vente est proposée pour 69 euros par mois ou pour une valeur finale de 3040 euros. Deux pièces sont installées et louées à l'administration du Palais de Tokyo à Paris de 2001 à 2003. Soussan Ltd réalise également une fontaine roulante, Donneuse d'eau, pour l'inauguration du Centre d'art de Pougues-les-Eaux le 12 décembre 1998, en référence à l'histoire de la station thermale.

- Enfin, le Porteur spécial est un service de messagerie par coursier à pied, effectué par l'artiste-marathonien Paul Robert et estampillé « no petrol $"{ }^{18}$. Du service/œuvre immatérielle, l'artiste propose respectivement un produit/multiple et un bon de commande/bon de souscription à une œuvre. En effet, l'énergie dépensée (6 volts à 0,5 milliAmpères par heure) est collectée et revendue sous forme d'accumulateurs de type R6 sur le site Internet pour 28 euros. En 2002, un bon de commande (" ticket courses: un service proposé par Paul Robert, coureur de fond soutenu par Soussan Ltd ») est édité pour l'exposition " La Cure, traitée d'art » contemporain au Centre d'art contemporain de Pougues-les-Eaux. 30 exemplaires sont offerts aux participants à l'exposition, transformée pour l'occasion en résidence pour curistes.

On constate que les activités de services, mises en place par Sylvain Soussan au sein de sa société, impliquent la production d'un certain 
matériel. Les objets qui en résultent sont des produits semblables à ceux de la grande distribution, ils en sont même souvent issus. Les accumulateurs R6, les gerbeurs, les diables, les isoloirs, les distributeurs de boisson et autres fontaines à eau sont des objets trouvés qui se voient simplement ajouter en impression le titre de l'œuvre et/ou le logo de Soussan Ltd. Comment l'application de l'emblème de la société d'un artiste sur des articles de consommation peut leur conférer des propriétés artistiques et faire d'eux des multiples en cas d'édition?

La réponse est dans le rapport étroit qu'entretiennent les objets avec la diffusion d'œuvres d'autres artistes, qu'elle soit immatérielle (message d'artiste-marathonien) ou matérielle (œuvres d'artistes présentées sur distributeurs, diffusées par diables ou déplacées par chariot élévateur). Il est en effet en adéquation avec l'intention de l'auteur, à savoir insérer ses productions dans un espace social donné: « Le détournement ne m'intéresse pas. Je tente d'utiliser les outils de production avec le maximum de présence d'esprit pour arriver à les employer différemment. Je veux travailler dans les cadres existants et voir comment il est possible de les aménage ${ }^{19}$ ». Autrement dit, en se mettant au service d'autres artistes, Sylvain Soussan rend visible sa production personnelle par le biais d'éléments matériels, parfois multipliés, et que l'on retrouve, de manière plus développée, dans le mécénat.

\section{Soussan Ltd: les produits d'appel du mécénat}

Le mécénat est le soutien moral apporté par Soussan Ltd. en échange d'un bénéfice publicitaire. Il comprend le sponsoring de la galerie le Sous-Sol et d'industriels (Kiss, Tollens, Ruget, Fuji Film...) à partir de 1994, du coureur de fond Paul Robert et du triathlète Cyril Delage (tee-shirts, sweat-shirts, boissons, casquettes estampillés du logo Soussan Ltd, à l'usage exclusif des deux sportifs) et la création de la Fondation Soussan Ltd à l'époque des grands travaux afin d'offrir une dimension de prestige à l'entreprise et d'en asseoir une crédibilitée ${ }^{20}$. Cette fondation conserve la collection de peintures du $19^{e}$ siècle de la société et est chargée d' " encourager la création des musées en redistribuant des fonds pour le montage d'expositions temporaires, la production audiovisuelle et l'édition ${ }^{21}$ ".

Dans ce cadre, la fondation Soussan Ltd produit des éditions, à l'instar du cadeau d'entreprise, Mémoire, offert avec le concours de la marque Gerblé. Au total, quatre cents barres ont été distribuées gracieusement aux visiteurs lors d'un vernissage au Musée d'art moderne de la Ville de Paris le 20 septembre 1995. Cette distribution
19 Sylvain Soussan, entretien avec Emmanuelle Chérel, op. cit., p. 23.

${ }^{20}$ La fondation est née de la nécessité de conserver un patrimoine immobilier. Tous les tableaux existent, une quinzaine sont d'origine mystérieuse, mais la fondation n'a pas de statut officiel.

${ }^{21}$ Sylvain Soussan, Soussan Ltd. Fournisseur des musées, Sylvain Soussan (dir.), Savignyle-Temple, Écomusée; Meymac, Abbaye SaintAndré Centre d'art contemporain, 1991, n.p.

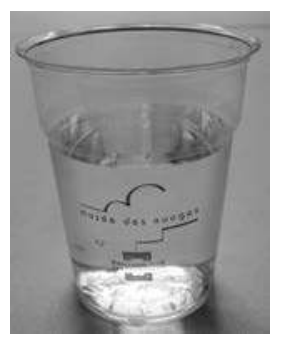


${ }^{22}$ Cinquante bouteilles de la même marque (" Conservez votre instinct de conservation ») ont été également distribuées au dîner d'inauguration des expositions de Louise Bourgeois et Claude Cahun au Musée d'art moderne de la Ville de Paris en juin 1995. est accompagnée de la diffusion d'un tract. Sylvain Soussan a souhaité faire une campagne de publicité sur la mémoire auprès du public. L'échantillon diététique met en évidence l'état transitif de la consommation qu'elle soit objet usuel ou objet d'art.

Dans le même esprit, le mécénat intègre le Musée des nuages, une association à but non lucratif, créée sur l'initiative de la Fondation Soussan Ltd. Inauguré en 1990 pour l' "Exposition n ${ }^{\circ} 1$ » à Belleville-sur-Loire, ce musée traite de la problématique de l'eau et de l'écologie. Comme l'évoque son logo, cette institution culturelle expose en général des nuages. Sylvain Soussan souhaite voir le musée ouvert à la société et situé au cœur de la nature. De ce fait, il diffuse des produits qui affirme l'intérêt du Musée des nuages pour les patrimoines naturels ainsi que la prise en compte de la satisfaction du public et des médiateurs de l'art.

Certains produits appartenant à une installation ne font ni l'objet d'édition ni d'appropriation plurielle. Destinés à un seul acquéreur, ils ne constituent donc pas des multiples. C'est le cas des Abreuvoirs d'eau de source destinés aux oiseaux et installés près du pavillon des sources taries du Parc Saint-Léger lors de l'exposition "Séjours 98 » à Pougues-les-Eaux. À l'inverse, les deux cents bouteilles d'eau Contrex en verre avec un surétiquetage (" Boire est un acte naturel, c'est aussi un comportement culturel »), distribuées aux galeries de la FIAC 1994 sont des multiples à part entière22.

Au-delà de cette diffusion dans le cadre de manifestations artistiques, la marque du Musée des nuages trouve sa place sur des produits manufacturés, récupérés par l'artiste qui y appose simplement son logo. Ici encore, certains produits servent uniquement au développement promotionnel d'un espace et ne peuvent pas faire l'objet d'une acquisition. C'est le cas du cendrier installé à l'entrée de l'exposition itinérante «Art \& Tabac » lancée au Palazzo Ruspoli en 1993 ou des deux pavillons hissés lors de l'exposition « L'Art dans le vent » au Domaine de Chamarande en 2000.

En revanche, les produits édités et distribués aux personnes intéressées ou aux contacts de l'artiste, sont des multiples. On mentionnera l'édition des calendriers-cartes de visite diffusés entre 1996 et 2000 , celle des gobelets de pique-nique transparents distribués autour d'une piscine à l'École Nationale d'Aviation Civile de Toulouse en 2003 (1000 exemplaires) ou encore celle des verres à limonade IKEA utilisés pour un cocktail au bar de la Biennale de Paris 2004 (50 exemplaires). Les produits édités et diffusés par l'intermédiaire de comptoirs de vente sont aussi concernés. Le premier, installé à l'Écomusée de Savigny-le-Temple en 1990, met par exemple en vente des diagrammes d'un hygromètre ou thermohygrographe, 
des autographes de Marcel Soussan ou encore des disquettes informatiques avec le label Soussan Ltd. Un autre, à la galerie Le Soussol en 1996, propose notamment un rouleau de peinture avec le logo de Soussan Ltd (3 exemplaires) et une plaquette publicitaire « originale » en blanc sur la vente organisée par Daniel Walravens et Bernard Brunon (500 exemplaires).

Ce qui frappe dans cette activité de mécénat, c'est le développement des éditions face aux œuvres uniques ou restées à l'état de prototypes, plus nombreuses dans les secteurs des produits et des services de Soussan Ltd. Il est rendu possible par la seule application du logo de la société ou de sa filiale du Musée des nuages sur des objets manufacturés et le recours à l'imprimé plus facilement reproductible. Si ces productions font également l'objet d'un tirage notable, c'est parce qu'elles visent - selon Sylvain Soussan - non seulement à vulgariser l'art au sens pédagogique du terme, mais aussi à promouvoir l'entreprise. Elles sont en effet diffusées comme cadeaux de fin d'année ou comme " appâts ». L'artiste donne l'exemple de l'envoi d' " hameçons publicitaires » à des conservateurs ${ }^{23}$.

Cette fabrication s'inscrit dans le contexte du développement d'un secteur en expansion: celui de la « paramuséologie » - ensemble des boutiques, restaurants, produits et services dérivés présents dans le cadre des musées. En calquant le système de l'entreprise par la proposition de produits et de services à destination des musées et d'autres institutions culturelles, Sylvain Soussan ne peut omettre l'instauration de cette activité marchande parallèle. Les objets qui y sont diffusés adoptent donc un fonctionnement promotionnel. Et pourtant, en s'inscrivant dans une appropriation stratégique du système de l'entreprise pour le replacer dans un cadre culturel, ses produits d'appel du mécénat n'en constituent pas moins des multiples à part entière, au même titre que les éditions issues des secteurs des produits et des services.

Il résulte de ce qui précède qu'en s'inspirant d'un système économique existant, Sylvain Soussan met en place sa propre entreprise qui, par la matérialisation, la reproductibilité et la diffusion d'un certain nombre d'éléments contribuent à installer la pratique du multiple.

Pour cela, il utilise plusieurs éléments propres aux unités commerciales extérieures à l'art afin de diffuser ses propositions émanant des différents secteurs (produits, services, mécénat) de son entreprise. Ainsi, les objets réalisés imitent des biens de consommation courante. L'artiste emprunte également à la publicité ses tactiques commerciales (logotypes sur supports de communication), ses
${ }^{23}$ Sylvain Soussan, entretien avec l'auteur, 2005. 
réseaux de diffusion (tracts) et ses actions (coursier). Enfin, il utilise les équipements et les modes de distribution du système commercial traditionnel (vente en ligne).

Cependant, cette transposition est relativisée tant sur le plan esthétique que sur le plan pratique par différentes modalités, du reste souvent complémentaires. D'abord, les objets proposés marquent eux-mêmes leur distance avec les biens de consommation, à l'instar des « outils-faits-main-pour-votre-main » réalisés en matériaux précieux. La fabrication en série permet cette mise à distance du modèle. Elle est d'ailleurs loin d'être systématique ou calquée sur celle de l'industrie. En outre, la diffusion des œuvres demeure souvent dépendante d'un réseau commercial de l'art (présentation dans les galeries ou les musées) et ce justement parce que l'intérêt esthétique des propositions de Sylvain Soussan réside dans le maintien de ce rapport entre les objets produits et le contexte muséal dans lequel ils interviennent.

Il apparaît donc que les propositions esthétiques multipliées, réalisées au sein de l'entreprise Soussan Ltd, présentent des formes à la fois d'ajustement et de résistance aux modèles sociaux et économiques qu'elles empruntent. Elles mettent en évidence la distance qui les maintient dans une production et une économie de l'art et le multiple y joue un rôle notable.

En effet, c'est le multiple qui permet non seulement de concrétiser la production matérielle issue de cette société du tertiaire mais aussi de faciliter l'accès des personnes intéressées à une forme d'art, parfois pour un moindre coût, voire gratuitement. Dans le même temps, c'est le multiple qui dissuade une certaine expansion de cette même production, de par sa dimension artistique, dépendante ici d'un contexte artistique dominant dans le dispositif global mis en place par l'artiste.

\section{Océane Delleaux}

